\title{
Optimizing ICA in fMRI using information on spatial regularities of the sources
}

Citation for published version (APA):

Valente, G., de Martino, F., Filosa, G., Balsi, M., \& Formisano, E. (2009). Optimizing ICA in fMRI using information on spatial regularities of the sources. Magnetic Resonance Imaging, 27(8), 1110-1119. https://doi.org/10.1016/j.mri.2009.05.036

Document status and date:

Published: 01/01/2009

DOI:

10.1016/j.mri.2009.05.036

Document Version:

Publisher's PDF, also known as Version of record

Document license:

Taverne

Please check the document version of this publication:

- A submitted manuscript is the version of the article upon submission and before peer-review. There can be important differences between the submitted version and the official published version of record.

People interested in the research are advised to contact the author for the final version of the publication, or visit the DOI to the publisher's website.

- The final author version and the galley proof are versions of the publication after peer review.

- The final published version features the final layout of the paper including the volume, issue and page numbers.

Link to publication

\footnotetext{
General rights rights.

- You may freely distribute the URL identifying the publication in the public portal. please follow below link for the End User Agreement:

www.umlib.nl/taverne-license

Take down policy

If you believe that this document breaches copyright please contact us at:

repository@maastrichtuniversity.nl

providing details and we will investigate your claim.
}

Copyright and moral rights for the publications made accessible in the public portal are retained by the authors and/or other copyright owners and it is a condition of accessing publications that users recognise and abide by the legal requirements associated with these

- Users may download and print one copy of any publication from the public portal for the purpose of private study or research.

- You may not further distribute the material or use it for any profit-making activity or commercial gain

If the publication is distributed under the terms of Article $25 \mathrm{fa}$ of the Dutch Copyright Act, indicated by the "Taverne" license above, 


\title{
Optimizing ICA in fMRI using information on spatial regularities of the sources
}

\author{
Giancarlo Valente $^{\mathrm{a}, *}$, Federico De Martino ${ }^{\mathrm{a}}$, Giuseppe Filosa ${ }^{\mathrm{b}}$, Marco Balsi ${ }^{\mathrm{b}}$, Elia Formisano ${ }^{\mathrm{a}}$ \\ ${ }^{\mathrm{a}}$ Faculty of Psychology and Neuroscience, Department of Cognitive Neuroscience, Maastricht Brain Imaging Center, Maastricht University, \\ 6200 MD Maastricht, The Netherlands \\ ${ }^{\mathrm{b}}$ Faculty of Engineering, Department of Electronic Engineering, University of Rome “La Sapienza”, I 00184 Roma, Italy \\ Received 22 October 2008; revised 6 March 2009; accepted 10 May 2009
}

\begin{abstract}
Spatial independent component analysis (ICA) is a well-established technique for multivariate analysis of functional magnetic resonance imaging (fMRI) data. It blindly extracts spatiotemporal patterns of neural activity from functional measurements by seeking for sources that are maximally independent. Additional information on one or more sources (e.g., spatial regularity) is often available; however, it is not considered while looking for independent components. In the present work, we propose a new ICA algorithm based on the optimization of an objective function that accounts for both independence and other information on the sources or on the mixing model in a very general fashion. In particular, we apply this approach to fMRI data analysis and illustrate, by means of simulations, how inclusion of a spatial regularity term helps to recover the sources more effectively than with conventional ICA. The improvement is especially evident in high noise situations. Furthermore we employ the same approach on data sets from a complex mental imagery experiment, showing that consistency and physiological plausibility of relatively weak components are improved.
\end{abstract}

(C) 2009 Elsevier Inc. All rights reserved.

Keywords: Independent Component Analysis; Blind source separation; fMRI; Spatial regularity; Simulated annealing

\section{Introduction}

Independent Component Analysis (ICA) aims to find a linear decomposition of a given data set into a more "meaningful" one, by achieving maximal independence among the vectors of the new representation [1]. Since its introduction, this technique has received great attention in various research fields, due to the generality of the assumptions underlying its model. In fact, like other "blind" techniques, no specific assumptions are made on the nature of the independent sources or on the way they have been mixed to generate the observations.

ICA has been successfully employed in many research fields, including functional neuroimaging, where its data-

* Corresponding author. Faculty of Psychology and Neuroscience, Department of Cognitive Neurosciences, University of Maastricht, P.O. Box 616, 6200 MD Maastricht, The Netherlands. Tel.: +31 433882469; fax: +31 433884125 .

E-mail address: giancarlo.valente@psychology.unimaas.nl(G. Valente). driven nature makes it a powerful tool to extract relevant information from functional magnetic resonance imaging (fMRI) and electro- and magnetoencephalography (EEG/ MEG) data, without relying on strong a priori assumptions [2]. Since its initial application to fMRI data analysis by McKeown et al. [3,4], ICA has become a well-established analytical tool for functional neuroimaging. ICA is applied to fMRI data as an alternative to traditional hypotheses-driven techniques (e.g., General Linear Model), to reveal neural activity embedded in a complex background of noise and other (independent) activities. Furthermore, it helps highlight signal co-variations in networks of brain regions (functional connectivity).

Considering an $m$-dimensional random vector $\mathbf{x}$, the classical ICA model for the observation is:

$\mathbf{x}=\mathbf{A s}$

where $\mathbf{s}$ is an $n$-dimensional vector whose components are maximally independent and it is usually referred to as the sources vector, $\mathbf{A}$ is an $m$ by $n$ matrix, often called mixing 
matrix, that accounts for the mixing process of the sources. The aim of ICA is to retrieve both $\mathbf{A}$ and s "blindly," i.e., without making specific assumptions on the nature of the sources or of the mixing process. To solve an ICA problem, a linear transformation $\mathbf{W}$ on observed data is performed such that the estimated sources

$\mathbf{y}=\mathbf{W x}$

are maximally independent (thus $\mathbf{W}$ is an estimate of the unmixing matrix $\mathbf{A}^{-1}$ ).

Several criteria have been proposed in order to obtain independence including maximization of differential entropy (INFOMAX [5]) and maximization of a robust approximation of negative entropy (negentropy, FastICA [6]). For comprehensive reviews of ICA algorithms, see Refs. [7,8].

In many situations, some additional information on the sources is available, but it is generally discarded while performing ICA decomposition. There are several reasons to incorporate prior information in an ICA model, when it is available. In fact, if a particular feature of a source is known in advance, it is possible to retrieve it first, without extracting the whole data set of components and, thereafter, performing a post-selection. This could be extremely useful in real-time applications and when a deflation approach is employed, since errors which arise whilst retrieving a source propagate to the subsequently extracted ones, and therefore, the source may not be extracted satisfactorily [9]. Moreover, if the prior information is accurate, in a high-noise situation, it can be employed to improve source recovery.

Several algorithms and methodologies have been proposed in the literature to account for prior information on an ICA problem. In Ref. [10], a methodology named "Principal Independent Component Analysis" was developed in order to extract a target source first. This procedure is based on a cumulant approach, where the learning is driven by a reference generator based on prior information on the target source. In Ref. [11], the authors, starting from second-order methods [12], show how it is possible to extract first a source whose autocorrelation function is known a priori. A Bayesian framework for source separation has been proposed in Ref. [13], showing how it is possible to account for information on the mixing coefficients or on the probability density functions of the sources. A Bayesian framework for source separation was presented by Rowe in $[14,15]$ and applied to fMRI data analysis in [16] to determine the reference function from functional time series.

As both sources and mixing coefficients are unknown, some prior information could be available on mixing coefficients rather than on sources. In Ref. [17], it has been shown how to account for knowledge on the mixing coefficients in the most employed ICA algorithms, showing how the inclusion of such information improves the speed of the algorithms. In Ref. [18], a constrained ICA approach was developed. Starting from Lagrange multipliers theory, the authors set up a "constrained ICA" algorithm where independence is maximized with an additional constraint imposed a priori. This technique was applied to fMRI data analysis, where it is possible to include a reference function (i.e., an approximation of the time-course of an experimental condition) into temporal ICA, extracting therefore only task-related sources. This technique has been applied also in [19] to EEG and MEG data analysis, to remove artifacts. In Ref. [20], a complementary approach was implemented to include this information in spatial ICA by extracting all the sources together and performing a gradient optimization of independence together with a term influencing correlation between the selected source and the time course. The proposed approach, named "semi-blind ICA," has proved useful as an alternative to General Linear Model, whereas the prior information on the experimental paradigm lacks precision. In Ref. [21], a framework to include specific templates of spatial activation into spatial ICA was proposed.

All the proposed approaches, however, consider only specific knowledge on the sources. When there is some information available on an unknown group of sources, none of the proposed approaches can incorporate it into the ICA extraction procedure.
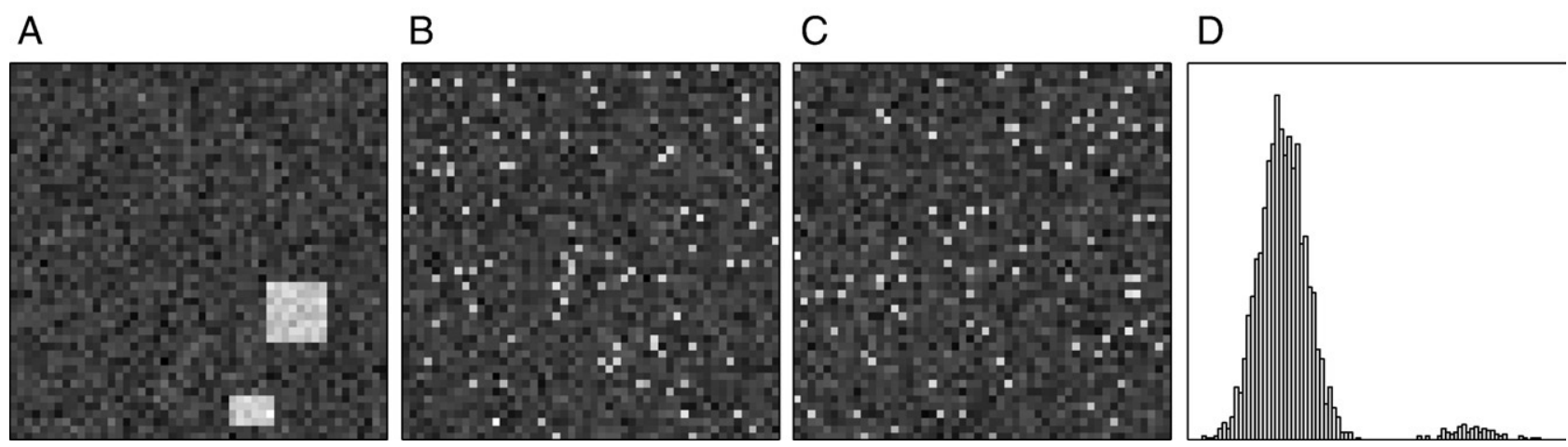

Fig. 1. Three different spatial maps (A-C) having the same histogram (D). An algorithm that only employs probability densities, may discard the information of spatial regularity in map (A). 
Consider fMRI data analysis; some more general assumptions, in addition to independence, can be made for the source recovery. A physiologically plausible activation obtained by ICA (in both spatial and temporal version) should show regularities in space and in time. However, classical ICA algorithms do not pursue this objective while looking for statistical independence. An illustrative example is presented in Fig. 1, where three 2dimensional maps have the same histogram, but completely different interpretation. In fact, the first one (a) may be interpreted as a meaningful activation, while the others $(b-c)$ carry no useful spatial information. In many of the common ICA algorithms employed in fMRI, data are always treated as "random variables" rather than "signals," meaning that only their joint statistical distribution is considered while performing extraction, regardless of the structure in space or in time.

In order to include a regularity term in the extraction, we have developed a new ICA approach. We employ an objective function, the optimization of which leads to the desired decomposition that accounts for both independence and prior knowledge.

\section{Materials and methods}

\subsection{ICA with prior information}

Our approach is based on the maximization of a new objective function defined as follows:

$F=J_{G}+\lambda H$

$J_{\mathrm{G}}$ is a negentropy estimate, as in [6], defined as:

$J_{G}(\mathbf{y})=[E\{G(\mathbf{y})\}-E\{G(\boldsymbol{\nu})\}]^{2}$

where $\boldsymbol{v}$ is a Gaussian distributed random variable with the same mean and variance as $\mathbf{y}$.

While $J_{\mathrm{G}}$ accounts for independence, $H$ is related to the available prior information and $\lambda$ weighs the two functions. The role played by the weight term $\lambda$ will be discussed extensively below.

If the additional term $\lambda H$ is set to zero, the maximization of $\mathrm{F}$ leads to "classical" independent components. As in FastICA algorithm, it is possible to perform independent component extraction in deflation and in symmetric approach. In the first one, sources are estimated one at a time, and each component is decorrelated from the previous ones during the extraction procedure. In symmetric approach, components are estimated all together, and the decorrelation procedure is applied at each iteration to the whole data set. After whitening, that linearly transforms original data set $\mathbf{x}$ into a new one $\tilde{\mathbf{x}}$ with identity covariance matrix, independent components can be retrieved from the unmixing coefficients matrix $\mathbf{Z}$ in the whitened space. For the deflation approach, Eq. (3) becomes:

$F(\mathbf{z})=J_{G}\left(\mathbf{z}^{T} \tilde{\mathbf{x}}\right)+\lambda H(\mathbf{z}, \tilde{\mathbf{x}})$

where $F, J_{G}$ and $H$ are real valued functions. For the symmetric approach, Eq. (3) becomes:

$F(\mathbf{Z})=\sum_{i} J_{G}\left(\mathbf{z}_{i}^{T} \tilde{\mathbf{x}}\right)+\lambda H(\mathbf{Z}, \tilde{\mathbf{x}})$

where $\mathbf{z}_{\mathbf{i}}$ denotes the $i$ th column of $\mathbf{Z}$. The decorrelation procedure for both cases is the same as in [6]. As pointed out in [1], an objective function suitable for an ICA problem must be invariant up to scale change, due to the double indeterminacy. This influences the possible choices of $H$, since it must be: $H(a \mathbf{z})=H(\mathbf{z}), \forall \mathbf{z} \neq 0$. However, since data are whitened, the unit norm constraint on independent components implies that $\|z\|^{2}=1$; therefore, $\mathrm{H}$ must be such that $H(-\mathbf{z})=H(\mathbf{z}), \forall \mathbf{z} \neq 0$.

The additional term $H$ poses new challenges to the optimization. In fact, in the FastICA algorithm, fixed-point iteration can be extremely fast thanks to a particularly convenient approximation of the Hessian matrix. Unfortunately, the inclusion of the additional term may be troublesome for a gradient-based scheme, in case it is not differentiable or defined in closed form. In order to also optimize these cases, we decided to perform optimization by means of simulated annealing (SA) [22] that allows dealing with global nondifferentiable maximization (minimization) by means of a random search with a probabilistic acceptance criterion. It has to be noted, however, that a SA optimization procedure is considerably slower than a gradient based one. Other optimization algorithms can be employed, according to the class of functions used for the objective function. The use of SA stems from the need for non-differentiable, or procedurally defined, objective functions, that cannot be optimized by employing derivatives. Another advantage of such an approach is that relatively minor modifications are needed when changing the objective function.

The core of a SA optimization is the Metropolis algorithm. Starting from a random state (i.e., a random choice of unmixing coefficients $\mathbf{w}_{0}$ ), by means of a suitable perturbation rule the system, gets to a new state $\mathbf{w}_{1}$ with associated cost function $F_{1}=F\left(\mathbf{w}_{1}\right)$. If $F_{1}>F_{0}=F\left(\mathbf{w}_{0}\right)$, then the new state is accepted; otherwise, it is accepted with a probability related to a control parameter $T$, often called temperature, evaluated as:

$p=\exp \left(\left(F_{1}-F_{0}\right) / T\right)$

SA is based on multiple iterations of the Metropolis scheme at different temperatures. The system starts from a high temperature (i.e., such that most of the perturbations are accepted anyway) and, according to a cooling schedule, decreases the temperature until the system "freezes" and 
reaches the optimum. Thanks to the probabilistic acceptance criterion, the algorithm reaches the global optimum with Probability 1, since it avoids getting trapped in local optima of the problem.

As discussed in [23], several perturbation schemes may be adopted in order to reach convergence. In our work, we implement the following rule (for the deflation scheme):

$\mathbf{w}_{k+1}=\mathbf{w}_{k}+\left(k_{0}+k_{1} / l\right) \boldsymbol{\delta}_{m}$

where $k_{0}$ and $k_{1}$ are some suitable constants, $l$ is a quantity that increases according to the temperature and $\boldsymbol{\delta}_{m}$ is an $m$ dimensional vector whose components are uniformly distributed $(-1,1)$. In this study, the values of the parameters $k_{0}$ and $k_{1}$ were 0.01 and 0.05 , respectively. Subsequently, we normalize the standard deviation and decorrelate the state $\mathbf{w}_{k+1}$ from the one generated by the previously found component before evaluating the objective function in the current state. A similar scheme is adopted for the symmetric extraction.

The annealing schedule (the combination of the temperature decrease rate and the number of iterations at each temperature) is crucial for the convergence of the algorithm, and several studies have been conducted in order to assess the convergence to the global optimum of the procedure [24]. Several annealing schedules have been proposed [25], and we choose to perform a geometric decrease rate (i.e., $T_{\mathrm{k}+1}=\alpha T_{\mathrm{k}}$ ) for the temperature, with $\alpha=95$. The starting temperature $T_{0}$ was automatically selected in such a way that, at $T_{0}$, the perturbations that were accepted were between $80 \%$ and $95 \%$ of the total.

\subsection{Spatial regularity term}

In this section, we show how to exploit generic prior information by means of an additional term in the classical objective function, whose aim is to improve source recovery, especially in noisy environments. This kind of information is general, in the sense that it is not related to a specific source but rather to a group of "interesting" and unknown sources. In particular, it is known that spatiotemporal patterns of activity exhibit regularities over both space and time. The most employed techniques for fMRI data analysis, namely FastICA and Infomax [26], completely discard this information, as they only employ joint statistics. In fact, if one scrambled all the voxels (in the same way) in each fMRI volume, the spatial independent component extraction would be exactly the same (i.e., with the same unmixing coefficients). However, information is present in a spatial map, as an independent component with isolated active voxels may have no physiological meaning. It is useful to exploit this "regularity" information together with independence in order to achieve an extraction where sources are more biologically plausible (like in Fig. 1A). It is to be noted that spatial regularity alone does not allow extracting sources. We therefore employed the scheme presented in the previous section to address this problem in spatial ICA, by optimizing independence together with a spatial regularity measure. The measure that proved the most effective is the one-lag spatial autocorrelation of the peak values of a spatial map. This autocorrelation is higher for those maps that present structured activity, and therefore, it can be seen as a measure of how plausible the map is, from an interpretative point of view. Therefore, adding this term to negentropy leads to less independent (that is, with lower values of negentropy) but more plausible solutions. It is to be noted, however, that the sources are still uncorrelated, as the source extraction is performed on the whitened data.

The evaluation of the additional term $H$ can be divided in two steps: spatial map thresholding and spatial autocorrelation evaluation. Concerning the first step, we consider the linear combination of the whitened data $\tilde{\mathbf{x}}$ by means of unmixing coefficient $\mathrm{z}, \mathbf{y}=\mathbf{z}^{T} \tilde{\mathbf{x}}$; since this linear combination is performed in the whitened space, it holds $\mu_{\mathrm{y}}=0$ and $\sigma_{\mathrm{y}}^{2}=1$. We choose an appropriate threshold $t$ and consider $\tilde{\mathrm{y}}=f_{t}(\mathbf{y})$, with $f_{t}(y)$ defined as follows:

$f_{t}(y)=\left\{\begin{array}{lll}y & \text { if } & |y| \geq t \\ 0 & \text { if } & |y|<t\end{array}\right.$

This thresholding procedure is employed due to the fact that it is more robust and physiologically plausible to focus the autocorrelation analysis on the "active" areas of a spatial map, rather than focusing on the overall autocorrelation map. Since sources of interest in fMRI have a super-Gaussian distribution, $t=2$ (two times the standard deviation) proved a suitable choice for the following analyses. Before evaluating the spatial autocorrelation on the thresholded map, we perform $z$-score transform of the thresholded map $\tilde{y}$.

The second step of the procedure is the evaluation of the spatial autocorrelation. Even if a spatial map is usually considered as a single vector, the spatial structure is intrinsically three-dimensional (two-dimensional if we restrict the analysis to a single slice or the reconstructed cortical sheet). In a simple monodimensional case, the onelag autocorrelation of a vector $\mathrm{T}$ can be evaluated as $H=E\{\mathbf{y}(t) \mathbf{y}(t+1)\}$, if the mean of $\mathbf{y}$ is zero. In a threedimensional problem, we consider all the possible translations in every direction and average them to have a global measure of autocorrelation. A computationally efficient way of evaluating such a measure, is to store in a matrix $\mathbf{N}$ the information on neighboring voxels. $\mathbf{N}$ is a highly sparse square matrix, with as many rows as voxels in the analysis, and where:

$N(i, j)=\left\{\begin{array}{cll}1 / K_{i} & \text { if } & i \in \mathbf{N}_{\mathrm{i}} \\ 0 & \text { if } & i \notin \mathbf{N}_{\mathrm{i}}\end{array}\right.$ 
where $\mathbf{N}_{\mathrm{i}}$ is the set of voxels that, within the cerebral cortex, is in the cube of edge length 3 (voxels) centered on voxel $i$, and $K_{\mathrm{i}}$ is a normalization constant, that accounts for the number of neighbors of each voxel and the total number of voxels in the cortical mask that are considered for the analysis. The spatial autocorrelation (when the mean of $\tilde{y}$ is zero) can be therefore evaluate in the following way:

$H(\tilde{\mathbf{y}})=\tilde{\mathbf{y}}^{T} N \tilde{\mathbf{y}}$

For the purposes of our analysis, we also considered a threshold applied to the spatial autocorrelation term, namely $t_{\mathrm{S}}$ such that it holds:

$H_{s}(\tilde{y})=\left\{\begin{array}{clc}H(\tilde{y}) & \text { if } & H(\tilde{y})<t_{s} \\ t_{s} & \text { if } & H(\tilde{y}) \geq t_{s}\end{array}\right.$

This thresholding procedure is motivated by the fact that maps that have an autocorrelation higher than $t_{\mathrm{s}}$ are already acceptable; therefore, no improvement is required in the autocorrelation term. The final objective function is:

$F\left(\mathbf{z}^{T} \tilde{\mathbf{x}}\right)=J_{G}\left(\mathbf{z}^{T} \tilde{\mathbf{x}}\right)+\lambda H_{s}$

It is to be noted that optimizing an objective function consisting of fastICA and an additional term that considers (thresholded) spatial autocorrelation, is not identical to extract sources from spatially smoothed data. As spatial filtering is a linear operation, the extracted sources of a smoothed data set are, under the ICA model, a smoothed version of the sources of the original data set, with identical mixing coefficients (see Ref. [7]). The proposed method, instead, improves estimation on both sources and mixing coefficient (as illustrated in Section 3). Furthermore, our method preserves the original spatial resolution of the data, with possible advantages for high spatial resolution functional imaging.

All the analyses presented in this work have been conducted using the fastICA package (http://www.cis.hut.fi/ projects/ica/fastica/) and a SA code developed for this work, both implemented in MATLAB (www.mathworks.com).

\subsection{Simulated fMRI data set}

We considered an fMRI resting state data set. It is well known from the literature (see, e.g., Ref. [27]) that during rest, some networks of underlying activity are still present and they can be found by means of spatial ICA. To evaluate the effectiveness of the source extraction procedure, we therefore superimposed some artificial activation, at different contrast to noise ratios. In this way it is possible to determine quantitatively how well a source is recovered in a realistic framework, as the artificial sources are embedded in a real fMRI data set. The contrast-to-noise ratio (CNR) is defined as the ratio between the signal enhancement $\Delta S$ due to activity, and the standard deviation of noise $\sigma_{n}$ in an active area [28]. We considered different situations, with values of CNR ranging from 0.8 to 3 , similar to Ref. [26].

The resting state experiment was performed by a healthy volunteer at the University of Maastricht, Department of Cognitive Neuroscience. The whole brain data was acquired
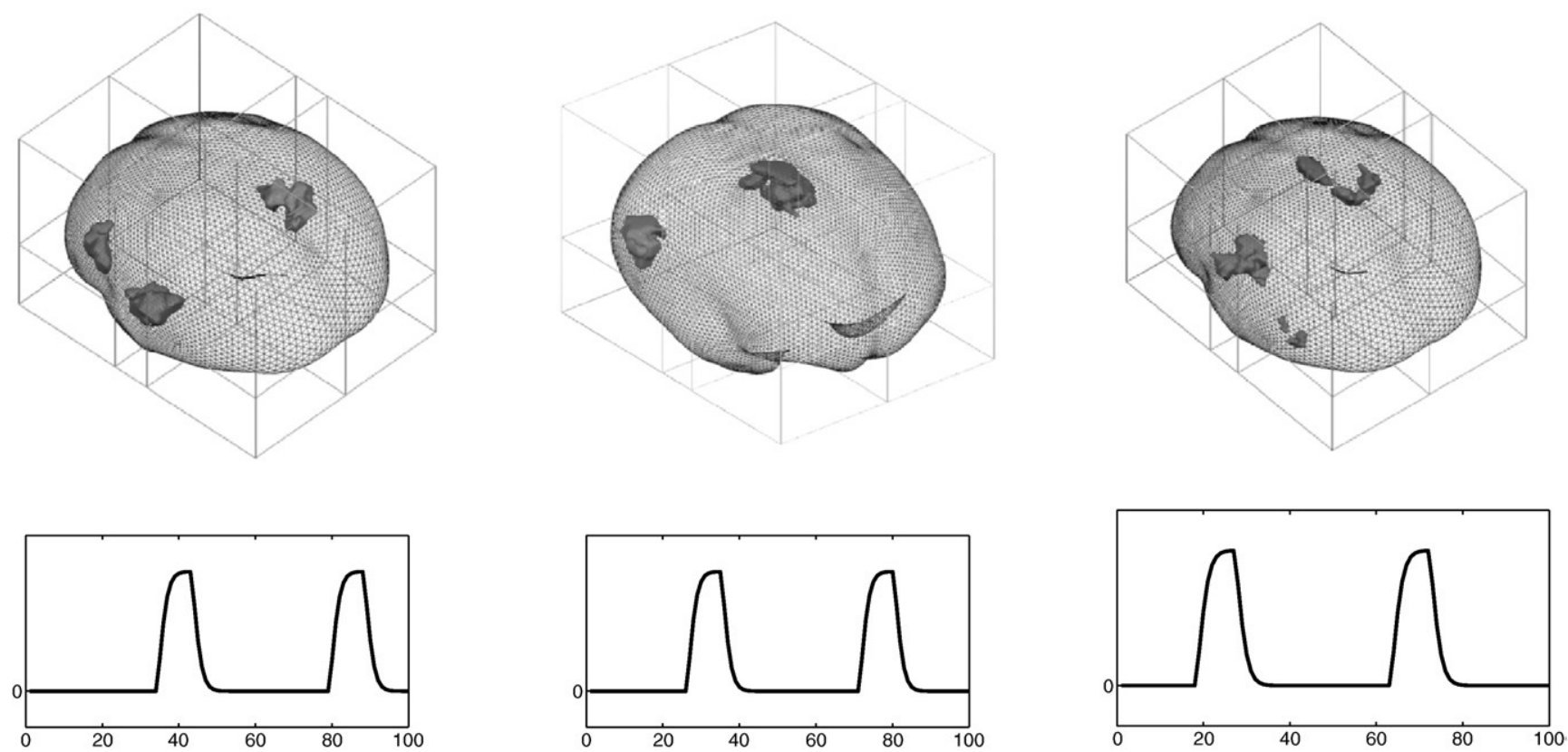

Fig. 2. Three artificial maps employed for the analysis on the simulated fMRI data set, together with respective time courses. The sources are injected into a resting state fMRI data set at different CNR ratios. Each source is visualized by means of a reconstructed glass brain view. 
on a 3 T Siemens Allegra (TR $1.5 \mathrm{~s}$, interslice time $46 \mathrm{~ms}$, 32 slices, matrix $64 \times 64$, thickness $3 \mathrm{~mm}, 210$ volumes). The first two volumes were skipped due to $\mathrm{T} 2 *$ saturation effect. Linear detrending and high pass filtering, removing low-frequency nonlinear drifts of three or less cycles $(0.009$ $\mathrm{Hz}$ ) per time series, were performed on the fMRI data set by means of a well-known fMRI data analysis tool, BrainVoyager QX (www.brainvoyager.com ). We performed cortex-based ICA [29], i.e., we reduced our analysis to only cortical voxels.

We considered three artificial activations, inserted on cortical sites with different time course (Fig. 2). Sources consisted of activations within: (a) (Source 1) left and right superior temporal gyrus and left inferior frontal gyrus (IFG); (b) (Source 2) right middle temporal gyrus and left and right inferior parietal sulcus; (c) (Source 3) left and right IFG (partially overlapping with source one) and left lateral occipital cortex. The time course of each of the three independent components has been obtained considering a square wave with ON and OFF periods of nine time points each. Each time course has been convolved with an estimate of the hemodynamic response function (HRF) [30], with parameters changing slightly from source to source, to account for a realistic variability of the HRF. We considered 20 transversal slices corresponding to Talairach transverse coordinate ranging from $z=114$ to $z=134$; in such a situation, the percentage of active voxels of the three artificial sources with respect to the total number of points considered for the analysis was $5.5 \%$, $4.9 \%$ and $4 \%$, respectively.

\section{4. fMRI experiment}

We furthermore considered a time-resolved fMRI experiment involving a complex mental imagery task [31]. Since complex tasks normally involve the activation of networks of brain regions with different HRFs, the use of a conventional hypothesis-driven method - assuming a spatially invariant model of the HRF - may not be optimal. ICA and other data-driven methods, instead, do not rely on explicit modeling of the hemodynamics and are thus particularly suitable in highlighting functional connectivity [32].

In the "auditory" condition of the experimental paradigm from Sack et al. [31], subjects were asked to build up simple geometrical figures based on a sequence of auditory instructions. They were subsequently presented a target figure and asked, after performing mental rotation, whether the imagined and target figures were identical or mirror symmetric versions of each other. During each functional run, 18 trials lasting $30 \mathrm{~s}$ each were acquired. The acquisition was performed on a 3-T Siemens Allegra scanner, using single-shot, gradient-echo, echo-planar imaging sequence (TE $30 \mathrm{~ms}$, TR $1500 \mathrm{~ms}$, matrix 64×64, 23 transversal slices, 730 images).

An analysis of these data using fuzzy clustering (FC) on the averaged time series revealed, besides other clusters, a set of interesting task-related clusters. This set of task-related clusters was very consistent within and across subjects and included one "auditory" cluster accounting for the responses to the auditory instructions, two "imagery" frontal-parietal clusters and a visual cluster that accounted for the response of the visual cortex to the presentation of the target. In particular, based on the relative timing of their time-courses, the two frontal-parietal clusters were labeled "Imagery early" and "Imagery late" and were interpreted as reflecting two subsequent cognitive processes involved in the mental imagery task (see Ref. [31] for details).

Using classical spatial ICA on the time series (without previous averaging as it was done for $\mathrm{FC}$ ) of the same data, we observed convergence in most of the subjects and found very similar spatiotemporal modes as the ones obtained with FC. However, in some "noisy" subjects, spatial ICA was not able to retrieve the "late" imagery cluster with frontalparietal distribution on all the runs [33]. In the present paper, we reevaluated these "noisy" data sets with the new algorithm including the spatial-regularity term. Based on the results of the simulations (see below), we expected an improvement, compared to FastICA, in retrieving the "weak" components both in terms of spatial layout and time course.

\section{Results and discussion}

\subsection{Simulated data set}

A comparison of classical ICA extraction (by means of FastICA) and the algorithm with the inclusion of the spatial regularity term was carried out on the simulated data set at different CNR levels. To evaluate and compare the two techniques, we employed receiver operating characteristic (ROC) analysis [26,34,35], estimating an ROC curve for each of the three injected sources recovery at each CNR level.

We performed an initial FastICA extraction on the resting state fMRI data set with superimposed activations at CNR 1 . We observed for each recovered source the values of negentropy and of spatial autocorrelation and considered the average among all the extracted components of the ratio $\rho=H_{s} / J_{g}$. We therefore decided to weight the additional term a half of the negentropy term. Since the average $\rho$ for the data set was approximately 10 , we set $\lambda$ to 0.05 . Furthermore, an appropriate choice for $t_{\mathrm{s}}$ was found to be $90 \%$ of the maximum value of spatial autocorrelation among the sources obtained with FastICA, namely 0.4. All the subsequent analyses have been carried out with these parameters. The employed annealing schedule consisted of a geometric temperature decrease rate with ratio $\alpha=0.8$, with a maximum number of iterations at a fixed temperature equal to 800 .

We conducted our analysis at different levels of CNR, ranging from 0.8 to 3 . For each level of noise, we performed 20 extractions for both algorithms. All data sets 

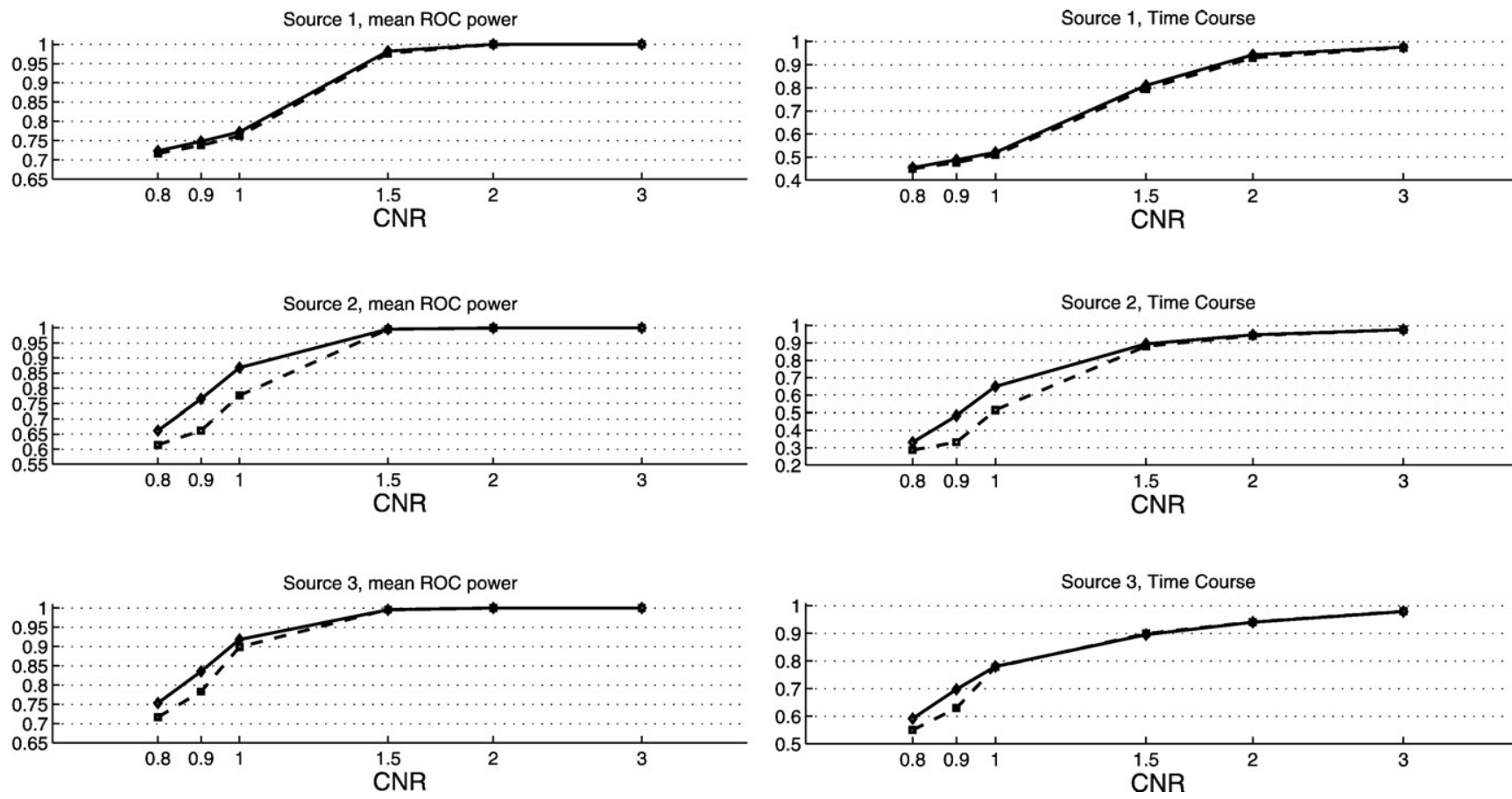

Fig. 3. Analysis of performances at different Contrast to noise ratios. FastICA values are indicated by a square (ם) symbol, ICA with spatial autocorrelation by a diamond $(\diamond)$ one. (Left) mean ROC power as a function of CNR. (Right) Correlation of the recovered time course with the original one. Results are presented in logarithmic scale with respect to $\mathrm{X}$ axis.

were composed of 100 volumes, to reduce computational load. Furthermore, no dimension reduction was carried out for both approaches, as in low CNR cases; this may affect source extraction for both algorithms. We therefore extracted 100 components for FastICA algorithm, while we stopped the sequential source extraction at the 60th component for our algorithm. This was due to the globaloptimum-driven nature of our optimization algorithm and to the observation that the three sources were always recovered among the first 40 components regardless of the CNR.

In Fig. 3 we considered the mean ROC power (i.e., the integral of the ROC curve) for the three sources and the two algorithms, at CNR ranging from 0.8 to 3 . Not surprisingly, in low-noise (high-CNR) situations, both algorithms are able to recover the sources with ROC power of nearly 1 , as seen in Fig. 3, left panel; in the right panel, instead, the correlation between the recovered time-courses and the original ones are depicted.

These results indicate that it is possible to enhance ICA capabilities by means of an additional term, which is not related to statistical properties of the sources, but to their spatial structure. One important remark is that, in low noise situation (i.e., high CNR), when negentropy alone is able to recover the sources satisfactorily, the additional term does not lead to any change in source extraction. On the other hand, when the noise level is such that classical algorithms performances deteriorate, the additional term helps improve the extraction.
The reason for the different behavior of the three recovering results for the three sources may be due to the fact that weight parameter $\lambda$ has been chosen in a heuristic fashion. Despite the good results obtained with Source 2, it may be possible that this parameter is less optimal with respect to Source 1, whose extraction is not significantly improved, and Source 3, where in low CNR situations, there is an improvement. These results suggest that further investigation in parameter setting must be done in order to assess the optimal parameter setting procedure. Nonetheless, a non optimal weight setting improves the source extraction procedure.

Another interesting issue is the noise level at which the proposed methodology (as well as classical ICA by means of FastICA) fail to reliably recover the sources of interest, but it is beyond the purpose of this work.

Table 1

Consistency of the retrieved components across different runs (subject "CJ")

\begin{tabular}{llllll}
\hline \multirow{2}{*}{ Component } & FASTICA & & \multicolumn{2}{l}{$\begin{array}{l}\text { Negentropy+spatial } \\
\text { regularity }\end{array}$} \\
\cline { 2 - 3 } \cline { 6 - 7 } & Mean & Variance & & Mean & Variance \\
\hline Auditory & 0.69 & 0.0047 & & 0.7383 & $3.7^{*} 10^{-4}$ \\
Imagery(I) & 0.5450 & 0.0215 & & 0.5817 & $8^{*} 10^{-4}$ \\
Visual/motor & 0.6517 & 0.0049 & & 0.6433 & 0.0048 \\
Imagery(II) & 0.3533 & 0.0546 & & 0.4450 & 0.0168 \\
\hline
\end{tabular}

For each of the techniques, the average and the variance of the spatial correlation are presented. 


\subsection{Real data set}

In the analysis of data from the mental imagery experiment, we performed a preliminary extraction by means of FastICA for each run. The setup of the objective function parameters ( $\lambda$ and $t_{\mathrm{s}}$ ) has been carried out for each run separately. The value of $\lambda$ was set according to the values of negentropy and spatial autocorrelation as in the simulated data set. The annealing schedule as well was the same employed for the simulated data set. We furthermore considered a threshold $t_{\mathrm{s}}$ equal to $95 \%$ of the highest spatial autocorrelation among the components found by means of FastICA.

We performed a comparison between the two approaches examining the task-related components across the different runs. All the components were manually inspected and labeled as referring to a particular cognitive processing stage (for each processing stage, we chose a single component). Subsequently, correlation between the spatial maps related to the same processes in different runs was evaluated. We considered the mean value of this spatial correlation (and its variance) for both FastICA and our approach.

The results on components related to the three main cognitive stages (auditory processing of the instructions, imagery of shapes and visual/motor responses to visual targets) are presented in Table 1, together with the results on the second Imagery component. These results indicate that the inclusion of the spatial autocorrelation term within the objective function increases (in a completely blind fashion) the consistency of the physiologically related independent components across the different runs.

Fig. 4 shows the time course of the second imagery component, averaged with respect to the experimental protocol, in the four runs and for both techniques, while the spatial layout of the maps is presented in Fig. 5. As already pointed out in [33], independence alone did not succeed in retrieving the second imagery component in all the four runs, as it can be seen in Fig. 4, left panel. In some of the runs, in fact, the brain networks involved in the cognitive process associated with the considered mental task were grouped together with other areas not involved in the task (and thus the discrepancies in the averages of the four runs). The spatial regularity terms makes the extraction more robust, without considerably altering the consistent components and increasing the consistency of the "weak" ones (and physiological plausibility, as the averages are general similar to those that can be obtained with other techniques that make explicit use of protocol information), as suggested by the simulated data set.

\section{Conclusion}

In this work we have introduced a new procedure to incorporate prior information into ICA extraction. The proposed methodology relies on a modification of a classical ICA objective function. This technique is very general, and allows optimizing objective functions which are not differentiable, or defined in a procedural fashion, using a suitable optimization algorithm as SA.

We have shown how to account for "generic" prior information by including a spatial regularity term into ICA extraction, and we have shown that this term helps in improving source recovery in low CNR situations, while at high CNRs, it yields results similar to those of classical ICA. The effectiveness of the source recovery can be seen by
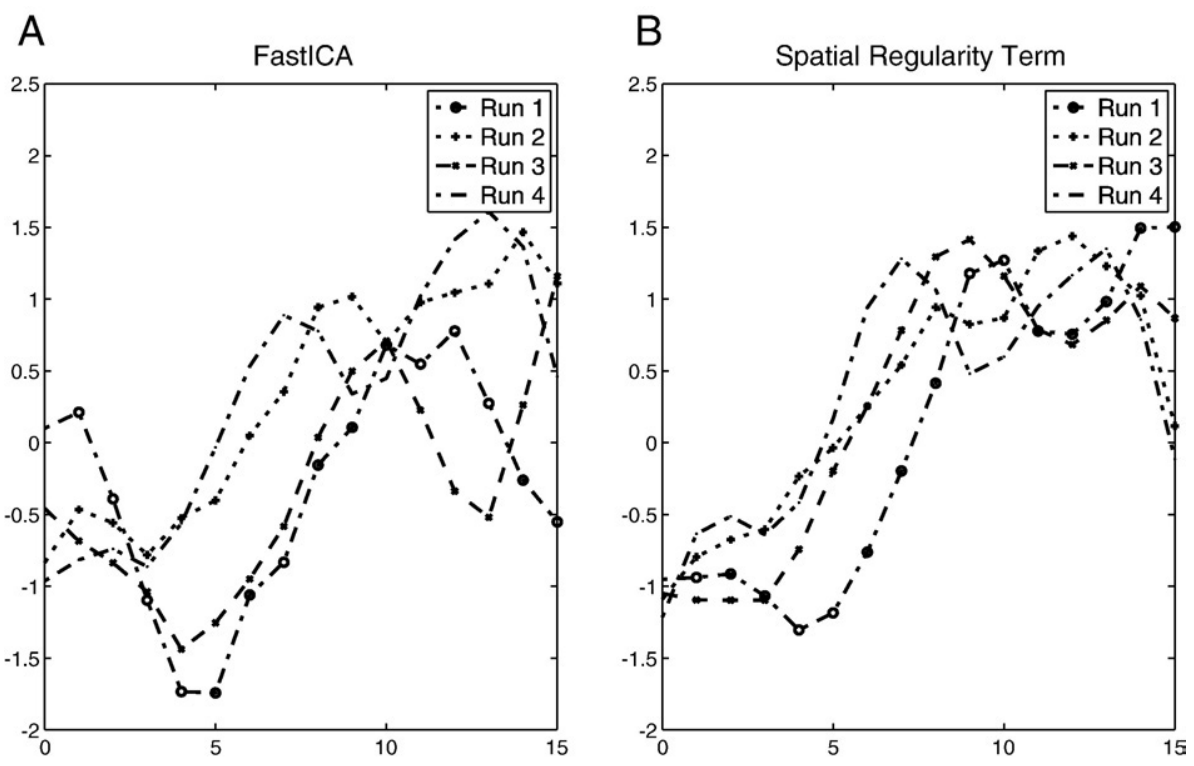

Fig. 4. Average of the time courses of the components associated with the second Imagery processing stage in the four runs of subject "CJ." The values are in normalized units. (A) FastICA extraction. (B) Negentropy with the additional spatial regularity term. 


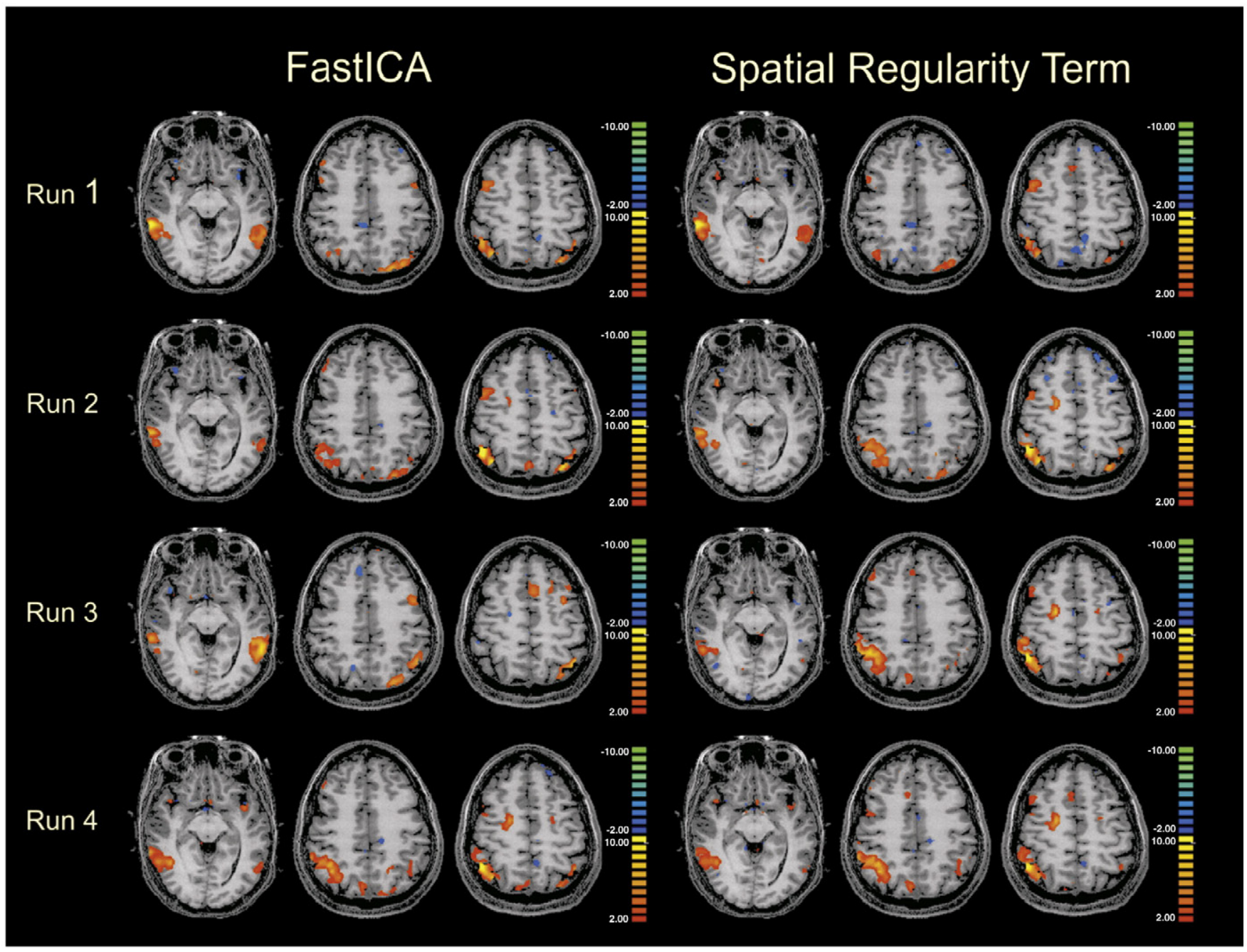

Fig. 5. Comparison between the retrieved independent components associated with the late imagery processing stage for the two algorithms in the four runs of the experiment.

means of ROC integrals and by the correlation of the recovered time courses with the original ones. Both these measures show that our methodology improves robustness of source extraction procedure to noise.

The same algorithm setting has been employed to analyze a time-resolved fMRI experiment, involving a complex mental imagery task. The results confirm the findings on simulated data set. In fact the additional term did improve the consistency of the retrieved components across runs, especially the weak ones, that independence alone did not prove able to recover in all the runs.

Further investigation is needed in order to extend these results to the analysis of data acquired with different field strength and acquisition parameters (e.g., spatial resolution).

\section{Acknowledgment}

Financial support from NWO (NWO-DFG grant DN 5519 and MaGW-VIDI grant 452-04-330) to E.F. is gratefully acknowledged. The authors would like to thank Marco Giordano for assistance during data analysis.

The authors are grateful to Martin Frost for comments on the manuscript.

\section{References}

[1] Comon P. Independent component analysis - a new concept? Signal Process 1994;36(3):287-314.

[2] Jung T, Makeig S, McKeown MJ, Bell A, Sejnowski TJ. Imaging brain dynamics using independent component analysis. Proc IEEE 2001;89 (7):1107-22

[3] McKeown MJ, Makeig S, Brown GG, Jung TP, Kindermann SS, Bell AJ, et al. Analysis of fMRI data by blind separation into independent spatial components. Hum Brain Mapp 1998;6:160-88.

[4] McKeown MJ, Sejnowski TJ. Independent component analysis of fMRI data: examining the assumptions. Hum Brain Mapp 1998;6: $368-72$.

[5] Bell A, Sejnowski TJ. An information-maximization approach to blind separation and blind deconvolution. Neural Comput 1995;7: $1129-59$ 
[6] Hyvärinen A. Fast and robust fixed point algorithms for independent component analysis. IEEE Trans Neural Netw 1999;10(3):626-34.

[7] Hyvärinen A, Karhunen J, Oja E. Independent component analysis. New York: John Wiley \& Sons; 2001.

[8] Amari S, Cichocki A. Adaptive blind signal and image processing learning algorithms and applications. New York: John Wiley \& Sons; 2002.

[9] Esposito F, Seifritz E, Formisano E, Morrone R, Scarabino T, Tedeschi $\mathrm{G}$, et al. Real-time independent component analysis of fMRI timeseries. Neuroimage 2003;20:2209-24.

[10] Luo J, Ling X, Liu R. Principal independent component analysis. IEEE Trans Neural Netw 1999;10(4):912-7.

[11] Barros A, Cichocki A. Extraction of specific signals with temporal structure. Neural Comput 2001;13(9):1995-2003.

[12] Belouchrani A, Meraim KA, Cardoso J, Moulines E. A blind source separation technique based on second order statistics. IEEE Trans Signal Process 1997;45(2):434-44.

[13] Knuth K. A Bayesian approach to source separation. In: Cardoso J-F, Jutten C, Loubaton P, editors. Proceedings of the First International Workshop on Independent Component Analysis and Signal Separation: ICA'99, Aussios, France; 1999. p. 283-8.

[14] Rowe DA. Bayesian approach to blind source separation. J Interdis Math 2002;5(1):49-76.

[15] Rowe D. Multivariate Bayesian statistics: models for source separation and signal unmixing. Boca Raton, FL, USA: CRC press; 2002.

[16] Rowe D. Bayesian source separation for reference function determination in fMRI. Magn Reson Med 2001;46:374-8.

[17] Igual J, Vergara L, Camacho A, Miralles R. Independent component analysis with prior information about the mixing matrix. Neurocomputing 2003;50:419-38.

[18] Lu W, Rajapakse J. Approach and applications of constrained ICA. IEEE Trans Neural Netw 2005;16(12):203-12.

[19] James C, Gibson O. Temporally constrained ICA: an application to artifact rejection in electromagnetic brain signal analysis. IEEE Trans Biomed Eng 2003;50(9):1108-16.

[20] Calhoun VD, Adali T, Pekar J, Pearlson G. Semi-blind I.C.A. of fMRI: a method for utilizing hypothesis-derived time courses in a spatial ICA analysis. Neuroimage 2005;25(2):527-38.

[21] Li YO, Adali T, Calhoun VD. A feature-selective independent component analysis method for functional MRI. Int J Biomed Imaging 2007;2007:15635.

[22] Kirkpatrick S, Gelatt CD, Vecchi MP. Optimization by simulated annealing. Science 1983;220(4598):671-80.
[23] Wah B, Wang T. Simulated annealing with asymptotic convergence for nonlinear constrained global optimization. Principles and Practice of Constraint Programming. International conference N.5, Alexandria, VA, USA. Berlin: Springer; 1999. p. 461-75.

[24] Aarts E, vanLaarhoven P. Simulated annealing: theory and applications. Dordrecht: Kluwer Academic Publishers; 1987.

[25] Atallah MJ, editor. Algorithms and theory of computation handbook. Boca Raton, FL: CRC Press; 1999. p. 1-19.

[26] Esposito F, Formisano E, Seifritz E, Goebel R, Morrone R, Tedeschi G, et al. Spatial independent component analysis of functional MRI timeseries: to what extent do results depend on the algorithm used? Hum Brain Mapp 2002;16:146-57.

[27] Van de Ven VG, Formisano E, Prvulovic D, Roeder CH, Linden DEJ. Functional connectivity as revealed by spatial independent component analysis of fMRI measurements during rest. Hum Brain Mapp 2004; 22:165-78.

[28] Baumgartner R, Ryner L, Richter W, Summers R, Jarmasz M, Somorjai R. Comparison of two exploratory data analysis methods for fMRI: fuzzy clustering vs. principal component. Magn Reson Imaging 2000;18:89-94.

[29] Formisano E, Esposito F, Di Salle F, Goebel R. Cortex-based independent component analysis of fMRI time-series. Magn Reson Imaging 2004;22(10):1493-504.

[30] Boynton G, Engel S, Glover G, Heeger D. Linear systems analysis of functional magnetic resonance imaging in human V1. J Neurosci 1996; 16(13):4207-21.

[31] Sack AT, Jacobs C, De Martino F, Staeren N, Goebel R, Formisano E. Dynamic premotor-to-parietal interactions during spatial imagery. J Neurosci 2008;28(34):8417-29.

[32] Formisano E, Linden D, Di Salle F, Trojano L, Esposito F, Sack A, et al. Tracking the mind's image in the brain: I. Timeresolved fMRI during visuospatial mental imagery. Neuron 2002; 35:185-94.

[33] Smolders A, De Martino F, Staeren N, Scheunders P, Sijbers J, Goebel $\mathrm{R}$, et al. Dissecting cognitive stages with time-resolved fMRI data: a comparison of fuzzy clustering and independent component analysis. Magn Reson Imaging 2007;25(6):860-8.

[34] Skudlarski P, Constable RT, Gore J. ROC analysis of statistical methods used in functional MRI: individual subjects. Neuroimage 1999;9:311-29.

[35] Fadili MJ, Ruan S, Bloyet D, Mazoyer B. A multi-step unsupervised fuzzy clustering analysis of fMRI time series. Hum Brain Mapp 2000; 10:160-78. 